\title{
Estudos experimentais sobre COVID-19: panorama da produção científica mundial
}

\author{
Experimental studies on COVID-19: overview of the world scientific production
} Estudios experimentales sobre COVID-19: panorama de la producción científica mundial

Livia Moreira Barros ${ }^{1}$ io https://orcid.org/0000-0002-0174-2255

Nelson Miguel Galindo Neto ${ }^{2}$ (I) https://orcid.org/0000-0002-7003-165X Guilherme Guarino de Moura Sá ${ }^{3}$ io https://orcid.org/0000-0003-3283-2656 Juliana de Castro Nunes Pereira ${ }^{3}$ io https://orcid.org/0000-0001-6831-1639

Luciana Uchôa Barbosa ${ }^{3}$ io https://orcid.org/0000-0002-3351-2075 Joaquim Guerra de Oliveira Neto ${ }^{4}$ io https://orcid.orgy/0000-0002-8068-2026 Amanda Haissa Barros Henriques ${ }^{5}$ io https://orcid.org/0000-0001-8735-225X

Joselany Áfio Caetano ${ }^{6}$ io hittps://orcid.org/00000-0002-0807-056X

\section{Como citar:}

Barros LM, Galindo Neto NM, Sá GG, Pereira JC, Barbosa LU, Oliveira Neto JG, et al. Estudos experimentais sobre COVID-19: panorama da produção cientifica mundial. Acta Paul Enferm. 2020;33:APE20200121.

DOI http://dx.doi.org/10.37689/actaape/2020A001215

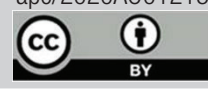

Descritores

Coronavirus; Infecções por coronavírus; COVID-19; Pandemias; Prática clínica baseada em evidências

Keywords Coronavirus; Coronavirus infections; COVID-19; Pandemics; Evidence-based practice

Descriptores

Coronavirus; Infecciones por coronavirus; COVID-19; Pandemias; Práctica clínica basada en la evidencia

Submetido 27 de Maio de 2020

Aceito

19 de Agosto de 2020

Autor correspondente Guilherme Guarino de Moura Sá E-mail: guilherme_mourasa@hotmail.com

\section{Resumo}

Objetivo: Descrever o panorama mundial da produção de estudos experimentais relacionados à COVID-19.

Métodos: Estudo descritivo, realizado em abril de 2020, a partir de busca pelos registros de ensaios clínicos, nos portais Clinical Trials e Registros Brasileiros de Ensaios Clínicos. A análise estatística foi descritiva.

Resultados: Dos 645 ensaios clínicos da amostra, houve predominância de 199 (30,9\%) oriundos da Europa, $213(33 \%)$ realizados por instituições hospitalares, $482(74,7 \%)$ com objetivo direcionado ao tratamento. Quanto às intervenções pesquisadas, $394(61,1 \%)$ foram sobre medicamentos; $70(10,8 \%)$ investigaram intervenções biológicas; 45 (7,0\%), intervenções com sangue e derivados; 40 (6,2\%), intervenções comportamentais; 38 (5,9\%), intervenções com equipamentos; 31(4,8\%), intervenções assistenciais/ procedimentais; 18 (2,8\%), intervenções para diagnóstico e nove $(1,4 \%)$, intervenções de suplementação dietética. Observou-se que, em $515(79,8 \%)$ a população estudada foi composta por adultos e idosos, 635 $(98,4 \%)$ investigaram ambos os sexos, o delineamento de $480(74,4 \%)$ incluiu randomização, de $482(74,7 \%)$ alocação paralela dos participantes e $373(57,8 \%)$ não possuíu o cegamento.

Conclusão: Os estudos experimentais sobre a COVID-19 foram oriundos da Europa, realizados por hospitais, sobre 0 tratamento em adultos e idosos, com randomização, mas sem cegamento. Os achados podem direcionar a realização de estudos, para contemplarem as lacunas identificadas.

\section{Abstract}

Objective: To describe the world panorama of the production of experimental studies on COVID-19.

Methods: Descriptive study conducted in April 2020, based on a search for clinical trial records on the Clinical Trials and Brazilian Clinical Trials Records portals. The statistical analysis was descriptive.

Results: Of the 645 clinical trials in the sample, there was a predominance of 199 (30.9\%) from Europe, $213(33 \%)$ performed by hospital institutions, $482(74.7 \%)$ with the objective aimed at the treatment. As for interventions surveyed, $394(61.1 \%)$ were on drugs; $70(10.8 \%)$ investigated biological interventions; $45(7.0 \%)$ interventions with blood and blood products; 40 (6.2\%), behavioral interventions; 38 (5.9\%), interventions with equipment; $31(4.8 \%)$, care/procedural interventions; 18 (2.8\%), diagnostic interventions and nine (1.4\%) dietary supplementation interventions. The studied population was composed of adult and elderly subjects in $515(79.8 \%)$ studies, 635 (98.4\%) investigated both sexes, the design of $480(74.4 \%)$ included randomization, of $482(74.7 \%)$ parallel allocation of participants and $373(57.8 \%)$ did not have blinding.

Universidade da Integração Internacional da Lusofonia Afro-Brasileira Redencão, CE, Brast.

Instituto Federal de Educação, Ciência e Tecnologia de Pernambuco, Pesqueira, PE, Brasil.

Instituto Federal de Educação, Ciência e Tecnologia de Pernambuco, Belo Jardim, PE, Brasil.

${ }^{4}$ Santa Casa de Misericórdia de Sobral, Sobral, CE, Brasil.

5Instituto Federal de Educação, Ciência e Tecnologia da Paraíba, João Pessoa, PB, Brasil.

${ }^{6}$ Universidade Federal do Ceará, Fortaleza, CE, Brasil.

Conflitos de interesse: nada a declarar. 
Conclusion: The experimental studies on COVID-19 originated from Europe, were conducted by hospitals, on treatment in adult and elderly subjects, with randomization but without blinding. The findings may direct the performance of studies addressing the identified gaps.

\section{Resumen}

Objetivo: Describir el panorama mundial de la producción de estudios experimentales relacionados con la COVID-19.

Métodos: Estudio descriptivo, realizado en abril de 2020, a partir de la búsqueda de registros de ensayos clínicos en los portales Clinical Trials y Registros Brasileiros de Ensaios Clínicos. El análisis estadístico fue descriptivo.

Resultados: De los 645 ensayos clínicos de la muestra, hubo predominancia de 199 (30,9\%) oriundos de Europa, 213 (33\%) realizados por instituciones hospitalarias, $482(74,7 \%)$ con objetivo orientado al tratamiento. Respecto a las intervenciones investigadas, 394 (61,1\%) fueron sobre medicamentos; 70 (10,8 \%) investigaron intervenciones biológicas; 45 (7,0\%), intervenciones con sangre y derivados; 40 (6,2\%), intervenciones de comportamiento; 38 (5,9\%), intervenciones con equipos; 31 (4,8\%), intervenciones asistenciales/procedimentales; 18 (2,8 \%), intervenciones para diagnóstico, y 9 (1,4\%), intervenciones de suplementos dietéticos. Se observó que en 515 ensayos (79,8 \%) la población estudiada fue compuesta por adultos y ancianos, en 635 (98,4 \%) se investigaron ambos sexos, el diseño de 480 $(74,4$ \%) incluyó aleatorización, de 482 (74,7 \%) asignación paralela de los participantes y 373 (57,8 \%) no poseían cegamiento.

Conclusión: Los estudios experimentales sobre la COVID-19 fueron oriundos de Europa, realizados por hospitales, sobre el tratamiento en adultos y ancianos, con aleatorización, pero sin cegamiento. Los resultados pueden orientar la realización de estudios que contemplen los vacíos identificados.

\section{Introdução}

A doença causada pelo coronavírus humano SARSCoV-2 (COVID-19), foi declarada como pandemia em 2020. Em maio de 2020, havia 5.404.512 casos e 343.514 mortes, distribuídos em 212 países. ${ }^{(1)}$

O surto de COVID-19 representa desafio para o sistema de saúde pública, na busca de estratégias que reduzam a ameaça clínica para a população. ${ }^{(2)} \mathrm{O}$ conhecimento sobre o agravo permanece limitado e com intuito de otimizar as ações de combate à pandemia, é relevante maior conhecimento acerca do SARS-CoV-2, ${ }^{(2,3)}$ a partir da realização de ensaios clínicos em larga escala. ${ }^{(3)}$

Ainda não existe conteúdo científico robusto para respaldar os protocolos terapêuticos ou vacinas específicas, de forma que diversos estudos experimentais estão em desenvolvimento, para avaliar a eficácia das opçóes de tratamento. ${ }^{(4,5)}$ Nesse contexto, os resultados das pesquisas experimentais possuem papel potencial para orientar o planejamento de intervençóes eficazes e, assim, modificar o desenho exponencial da trajetória da epidemia.

A assistência em saúde durante a pandemia possui mais chances de ser efetiva e segura se ocorrer respaldada por resultados de investigações científicas. Para que tais resultados sejam consultados pelos profissionais de saúde, é importante que as evidências sejam sintetizadas e apresentadas de forma descritiva. Mediante tal apresentação, será possível identificar temas poucos explorados nas pesquisas e métodos que não foram percorridos pelos estudos existentes. Dessa forma, os temas e métodos não explorados poderão ser alvo de pesquisas futuras, de forma a contribuir com o estado da arte acerca da temática.

Assim, o objetivo deste estudo foi descrever o panorama mundial da produção de estudos experimentais relacionados à COVID-19.

\section{Métodos}

Trata-se de estudo descritivo, quantitativo, realizado em abril de 2020, em dois portais online de registros de ensaios clínicos: o ClinicalTrials (www.clinicaltrials.gov), que consiste no maior portal de registros de ensaios clínicos administrado pelo National Institutes of Health, nos Estados Unidos, e o portal de Registros Brasileiros de Ensaios Clínicos (REBEC), disponível no site http://www.ensaiosclinicos.gov. $\mathrm{br} /$. A busca em ambos os portais foi efetuada por meio da utilizaçáo do descritor "COVID-19" no tópico condition ou disease, disponível no Medical Subject Headings (MeSH).

Os critérios de seleção foram: publicaçóes divulgadas em 2020, sem delimitação de idioma, e que abordassem estudos experimentais sobre a COVID-19. Foram excluídas pesquisas com registro duplicado ou que possuíam status de interrompido ou cancelado.

A partir da busca, foram encontrados 1093 registros de ensaios clínicos, dos quais 448 foram excluídos e 645 compuseram a amostra. A figura 1 apresenta o fluxograma de seleção dos estudos.

Foi utilizado instrumento estruturado, baseado no Consolidated Standards of Reporting Trials - 


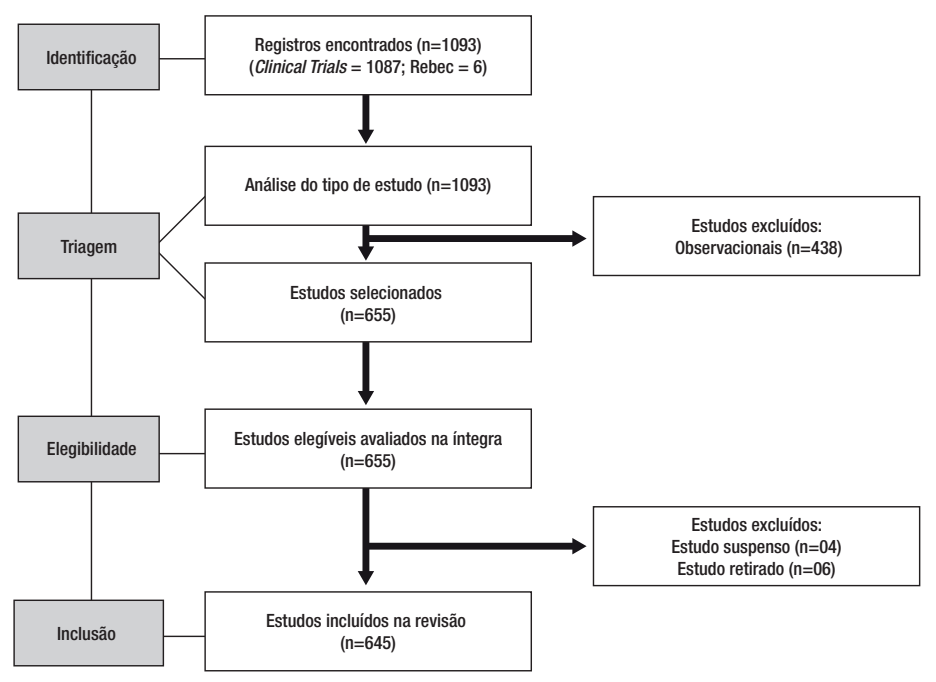

Figura 1. Fluxograma da seleção dos estudos

Consort 2010, para coleta de dados. As variáveis de interesse foram: país, população do estudo, critérios de elegibilidade para participantes (idade, sexo), assunto, tamanho de amostra, tipo de randomização e cegamento, alocação, tipo de intervenção, tempo de duração da coleta e informaçóes adicionais disponíveis no registro.

Foi realizado o acesso em cada registro disponibilizado nos portais para coleta das variáveis de interesse com auxílio do instrumento referido anteriormente. Ao final da coleta de todos os registros, variáveis como idade, tamanho da amostra, duração do estudo e país foram categorizadas para tornar a apresentação dos resultados mais objetiva.

Os dados foram tabulados no software Microsoft Excel 2010 e analisados estatisticamente por meio do software Statistical Package for the Social Sciences (SPSS), versão 24.0. Os resultados foram apresentados de forma descritiva com frequências absolutas e relativas. $\mathrm{O}$ estudo atendeu aos princípios éticos em pesquisa e não foi necessária submissáo em Comitê de Ética em Pesquisa com Seres Humanos, por tratar-se de estudo realizado com dados de domínio público.

\section{Resultados}

Dos 645 registros de ensaios clínicos analisados, $199(30,9 \%)$ eram provenientes da Europa, 178
(27,6\%) na América do Norte, 111 (17,2\%) não possuíam tal informação disponível, 109 (16,9\%) na Ásia, 29 (4,5\%) na América do Sul, 18 (2,8\%) na África e $1(0,2 \%)$ na Oceania. Em relação à instituição, 213 (33\%) possuíam colaboração de instituição hospitalar; 200 (31,0\%) de universidades; $146(22,6 \%)$ de hospitais universitários e 86 $(13,3 \%)$ de indústria farmacêutica.

No tocante ao status do recrutamento, 328 (50,9\%) ensaios clínicos não o tinham iniciado, 277 (42,9\%) haviam iniciado, 24 (3,7\%) encontravam-se em fase de envio de convite e 16 (2,5\%) haviam concluído o recrutamento. Referente ao objetivo, 482 $(74,7 \%)$ versavam sobre o tratamento; 87 (13,5\%) sobre a prevenção; $20(3,1 \%)$ contemplavam o cuidado de suporte; 16 (2,5\%), o diagnóstico; sete $(1,1 \%)$, pesquisa em serviços de saúde; cinco $(0,8 \%)$, a triagem e cinco $(0,8 \%)$ pesquisa básica.

O tempo de coleta de $306(47,4 \%)$ eram de até seis meses; em 187 (29,0\%), era de sete a 12 meses; em 110 (17,1\%), de um a dois anos e em 42 $(6,5 \%)$, superior a três anos. O tamanho da amostra teve mínimo de quatro e máximo de 55.000 participantes, com média de $681,81( \pm 3083,150)$ e mediana de 144 (intervalo interquartílico $=54.996$ ). A idade mínima dos participantes dos estudos foi de seis meses e a máxima foi de 104 anos. A caracterização dos ensaios clínicos, quanto aos assuntos abordados, randomização, tipo de alocação na in- 
tervenção, cegamento, tamanho da amostra e perfil dos participantes (sexo e ciclo vital), encontra-se detalhada na tabela 1 .

Tabela 1. Caracterização dos aspectos metodológicos dos ensaios clínicos sobre COVID-19

\begin{tabular}{|c|c|}
\hline Variáveis & $\mathrm{n}(\%)$ \\
\hline \multicolumn{2}{|l|}{ Assuntos } \\
\hline Tratamento medicamentoso & $394(61,1)$ \\
\hline Intervenções Biológicas & $70(10,8)$ \\
\hline Sangue e derivados & $45(7,0)$ \\
\hline Intervenções Comportamentais & $40(6,2)$ \\
\hline Uso de equipamentos & $38(5,9)$ \\
\hline Procedimento Assistenciais & $31(4,8)$ \\
\hline Testes para diagnóstico & $18(2,8)$ \\
\hline Suplementação Dietética & $9(1,4)$ \\
\hline \multicolumn{2}{|l|}{ Randomização } \\
\hline Randomizado & $480(74,4)$ \\
\hline Não-Randomizado & $56(8,7)$ \\
\hline Não informado & $109(16,9)$ \\
\hline \multicolumn{2}{|l|}{ Alocação na Intervenção terapêutica } \\
\hline Atribuição paralela & $482(74,7)$ \\
\hline Atribuição de grupo único & $124(19,2)$ \\
\hline Atribuição sequencial & $18(2,8)$ \\
\hline Atribuiç̧ão cruzada (crossover) & $12(1,9)$ \\
\hline Atribuição fatorial & $9(1,4)$ \\
\hline \multicolumn{2}{|l|}{ Cegamento } \\
\hline Aberto & $373(57,8)$ \\
\hline Único & $74(11,5)$ \\
\hline Duplo & $59(9,1)$ \\
\hline Triplo & $46(7,1)$ \\
\hline Quadruplo & $93(14,4)$ \\
\hline \multicolumn{2}{|l|}{ Tamanho da Amostra } \\
\hline 1 a 100 & $269(41,7)$ \\
\hline 101 a 500 & $251(38,9)$ \\
\hline 501 a 999 & $46(7,1)$ \\
\hline Acima de 1000 & $79(12,2)$ \\
\hline \multicolumn{2}{|l|}{ Sexo de Interesse } \\
\hline Ambos & $635(98,4)$ \\
\hline Somente feminino & $9(1,4)$ \\
\hline Somente masculino & $1(0,2)$ \\
\hline \multicolumn{2}{|l|}{ Ciclo vital dos Participantes } \\
\hline Crianças e adolescentes & $3(0,5)$ \\
\hline Adolescentes, adultos e idosos & $16(2,5)$ \\
\hline Apenas Adultos & $56(8,7)$ \\
\hline Adultos velhos e idosos & $7(1,1)$ \\
\hline Adultos e idosos & $515(79,8)$ \\
\hline Apenas Idosos & $14(2,2)$ \\
\hline Todo o ciclo vital & $34(5,3)$ \\
\hline
\end{tabular}

Observou-se que somente 6,8\% (44) dos estudos eram classificados como fase 4 , ou seja, aqueles que já possuem autorização de introdução no mercado. A maioria estava recrutando $(n=16 ; 36,4 \%)$ ou não recrutando participantes $(n=22 ; 50 \%)$. Esses estudos tinham como enfoque tratamento medicamentoso $(n=41 ; 93,2 \%)$, intervenções biológicas $(n=2 ; 4,5 \%)$ e suplementação dietéti- ca $(n=1 ; 2,3 \%)$. As principais intervençóes eram uso de cloroquina ou hidroxicloroquina $(n=17$; $38,6 \%)$, anticoagulantes como enoxaparina $(n=2$; $4,5 \%)$ e uso da vacina BCG para prevenção de COVID-19 ( $\mathrm{n}=2 ; 4,5 \%)$.

Os colaboradores eram instituiçóes hospitalares ou universidades com frequência de 40,9\% (18) em cada, principalmente da Europa $(n=21$; $47,7 \%)$ ou América do Norte $(n=10 ; 22,7 \%)$. O público-alvo eram adultos ou idosos $(n=36$; $81,8 \%)$ de ambos os sexos $(n=44 ; 100 \%)$. No que se refere às características metodológicas desses estudos experimentais, houve predomínio de amostra entre 1 a 100 participantes $(n=20$; $45,5 \%)$ ou 101 a $500(n=15 ; 34,1 \%)$. A maioria dos estudos apresentavam atribuição paralela em que dois ou mais grupos de participantes recebiam medicamentos diferentes $(n=32 ; 72,7 \%)$ e $56,8 \%$ (25) eram classificados como abertos.

Foi identificado o uso de 206 medicamentos, nos 394 estudos experimentais. Houve prevalência da Hidroxicloroquina em 78 $(19,76 \%)$, Hidroxicloroquina combinada com Azitromicina em 31 (7,85\%), combinação de Lopinavir e Ritonavir em 23 estudos (5,8\%), Azitromicina em 19 (4,8\%), Cloroquina em 16 (4\%), Tociliuzumab em 14 (3,5\%) e Ácido Ascórbico em 12 (3,0\%). Dos 125 estudos envolvendo o uso de cloroquina ou hidroxicloroquina, 40\% (50) estavam na fase 2 de ensaio clínico com predomínio de amostra entre 101 a 500 participantes $(n=51 ; 40,8 \%)$, randomiza$\operatorname{dos}(n=114 ; 91,2 \%)$ e abertos $(n=59 ; 47,2 \%)$. Os demais medicamentos foram variados, como Favipiravir, Colchicina, Metilpredinisolona, Ruxolitinib, Baricitinib, Sarilumab, Anakinra, Nitazoxanide e Remdesivir.

No tocante às intervençôes biológicas, a maioria comtemplou o uso de anticorpo monoclonal humanizado, enquanto, que, nas intervençôes com sangue e derivados, o plasma convalescente foi o mais utilizado. No tocante às intervençôes para diagnóstico, a identificação da sorologia de COVID-19 foi a mais prevalente. $\mathrm{O}$ detalhamento das intervençóes biológicas, com sangue e derivados e para diagnóstico, encontra-se na tabela 2 . 
Tabela 2. Distribuição das intervenções biológicas, com sangue e derivados e para diagnóstico, investigadas nos ensaios clínicos sobre COVID-19

\begin{tabular}{|c|c|}
\hline Variáveis & $n(\%)$ \\
\hline \multicolumn{2}{|l|}{ Intervenções biológicas ( $\mathrm{n}=70)$} \\
\hline Uso de anticorpo monoclonal humanizado & $25(35,7)$ \\
\hline Uso de células-tronco & $20(28,6)$ \\
\hline Uso de vacina para prevenção de COVID-19 & $14(20,0)$ \\
\hline Transferência de Células NK & $4(5,8)$ \\
\hline Suspensão bacteriana & $2(2,9)$ \\
\hline Autohemoterapia de Ozônio & $1(1,4)$ \\
\hline Amostras biológicas para correlação fenótipo/genótipo & $1(1,4)$ \\
\hline Fluido Amniótico Humano & $1(1,4)$ \\
\hline Sequenciamento Genoma & $1(1,4)$ \\
\hline Uso de anticorpo antiinterferon & $1(1,4)$ \\
\hline \multicolumn{2}{|l|}{ Intervenções com sangue e derivados ( $n=45)$} \\
\hline Uso de plasma convalescente & $32(71,2)$ \\
\hline Coleta de sangue para avaliar biomarcadores da COVID-19 & $4(8,9)$ \\
\hline Coleta de sangue para avaliar resposta imunológica & $2(4,5)$ \\
\hline $\begin{array}{l}\text { Coleta de sangue para avaliar hiperatividade do sistema renina- } \\
\text { angiotensina }\end{array}$ & $1(2,2)$ \\
\hline Coleta de sangue para avaliar hipercoagulação & $1(2,2)$ \\
\hline Coleta de sangue para avaliar soroconversão de profissionais de saúde & $1(2,2)$ \\
\hline Coleta de amostra de sangue para identificar Expressão do Receptor C5a & $1(2,2)$ \\
\hline Coleta de amostra de sangue para identificar neuromarcadores & $1(2,2)$ \\
\hline Uso de plasma hiperimune & $1(2,2)$ \\
\hline Coleta de sangue para avaliar Biomarcadores cardiovasculares e renais & $1(2,2)$ \\
\hline \multicolumn{2}{|l|}{ Intervenções para diagnóstico ( $n=18)$} \\
\hline Sorologia de COVID-19 & $5(27,8)$ \\
\hline Teste por PCR & $2(11,1)$ \\
\hline Comparação entre testes & $2(11,1)$ \\
\hline Teste rápido para COVID-19 & $2(11,1)$ \\
\hline Teste salivar & $2(11,1)$ \\
\hline Dosagem de citocinas & $1(5,6)$ \\
\hline Sensibilidade e especificidade entre testes & $1(5,6)$ \\
\hline Swab de células conjuntivais & $1(5,6)$ \\
\hline Swab de rinofaringe & $1(5,6)$ \\
\hline Ultrassom pulmonar & $1(5,6)$ \\
\hline
\end{tabular}

NK - Natural Killer; PCR- Reverse-transcriptase Polymerase Chain Reaction

Foram encontradas 31 intervençóes que envolviam utilização/testagem de equipamentos e 22 intervenções de caráter assistencial/procedimental. $\mathrm{O}$ posicionamento em prona foi identificado como principal intervenção assistencial (Tabela 3).

As intervençôes para suplementação dietética foram observadas em nove $(1,4 \%)$ ensaios clínicos, dos quais cinco $(55,6 \%)$ abordaram a utilização as vitaminas $\mathrm{C}$ e $\mathrm{D}$ e do zinco; e as intervençóes por dieta cetogênica voltada a pacientes intubados, uso de protótipos, uso de mel natural e de antioxidantes, foram abordados em um estudo (11,1\%), cada. As interven-
Tabela 3. Distribuição das intervenções com equipamentos e intervenções assistenciais/procedimentais investigadas pelos ensaios clínicos sobre COVID-19

\begin{tabular}{|c|c|}
\hline Variáveis & $n(\%)$ \\
\hline \multicolumn{2}{|l|}{ Intervenções com equipamentos ( $n=38)$} \\
\hline Dispositivos voltados à oxigenoterapia ${ }^{\mathrm{j}^{\star}}$ & $12(31,7)$ \\
\hline Dispositivos para procedimentos vasculares ${ }^{\dagger}$ & $8(21,2)$ \\
\hline Máscaras cirúrgica e N95 & $3(7,9)$ \\
\hline Sensor para monitorização de sinais vitais & $2(5,5)$ \\
\hline Plataforma de telereabilitação após a hospitalização & $1(2,6)$ \\
\hline Algoritmo para identificação de COVID-19 & $1(2,6)$ \\
\hline Barreira de contato durante endoscopia & $1(2,6)$ \\
\hline Caixa de barreira durante a intubação & $1(2,6)$ \\
\hline Calorímetro em pessoas com COVID-19 & $1(2,6)$ \\
\hline Colete V/Q na manipulação transtorácica & $1(2,6)$ \\
\hline Enxaguatório bucal & $1(2,6)$ \\
\hline Estimulador do nervo vago & $1(2,6)$ \\
\hline Inteligência artificial para auxiliar no ultrassom & $1(2,6)$ \\
\hline Protótipo de Swab & $1(2,6)$ \\
\hline Removedor extracorpóreo de dióxido de carbono & $1(2,6)$ \\
\hline Spray oral/nasal & $1(2,6)$ \\
\hline Termoterapia local & $1(2,6)$ \\
\hline \multicolumn{2}{|l|}{ Intervenções assistenciais/procedimentais $(n=31)$} \\
\hline Posicionamento Prona & $9(29,2)$ \\
\hline Ventilação mecânica & $3(9,8)$ \\
\hline Fisioterapia pulmonar & $2(6,6)$ \\
\hline Coleta de sangue para avaliar transmissão doméstica & $1(3,2)$ \\
\hline Eco Doppler para rastreio de trombose venosa profunda & $1(3,2)$ \\
\hline Intubação Pré-Hospitalar & $1(3,2)$ \\
\hline Avaliação do paciente após trombose venosa profunda & $1(3,2)$ \\
\hline Irrigação salina nasal & $1(3,2)$ \\
\hline Manobra de recrutamento alveolar & $1(3,2)$ \\
\hline Neuromodulação do ervo vago auricular & $1(3,2)$ \\
\hline Oxigenação por membrana extracorpórea (ECMO) precoce & $1(3,2)$ \\
\hline Ozonioterapia & $1(3,2)$ \\
\hline Reabilitação pulmonar & $1(3,2)$ \\
\hline Tomografia computorizada com autópsia minimamente invasiva & $1(3,2)$ \\
\hline Fração vascular estromal celular aut implantada por via intravenosa & $1(3,2)$ \\
\hline Oxigenoterapia hiperbárica & $1(3,2)$ \\
\hline Oxigenoterapia no isolamento domiciliar & $1(3,2)$ \\
\hline Reação em cadeia da polimerase (PCR) multiplex & $1(3,2)$ \\
\hline Radiação em baixa dose & $1(3,2)$ \\
\hline Administrador automatizado de oxigênio & $1(3,2)$ \\
\hline
\end{tabular}

"Câmara hiperbárica, válvula de oxigenação, dispositivos para treinamento respiratório, compressor automatizado para ventilação mecânica, laringoscópio, pressão positiva nas vias aéreas (CPAP), gerador hidrogênio/oxigênio com nebulizador, nebulizadores e cânula nasal de alto fluxo; ${ }^{\dagger}$ Adsorvedor de sangue para citocinas, Acesso intravascular, hemofiltro para membrana extracorpórea, adsorvedor de plasma, modulador de leucócitos

ções comportamentais mais frequentes foram referentes ao uso da telemedicina para monitoramento domiciliar, apoio psicossocial online e vídeos educativos para orientação de exercícios físicos (Tabela 4). 
Tabela 4. Distribuição das intervenções comportamentais dos ensaios clínicos sobre COVID-19

\begin{tabular}{lc}
\hline Variáveis & $\mathbf{n}(\%)$ \\
\hline Intervenções Comportamentais ( $\mathrm{n}=40$ ) & \\
Monitoramento domiciliar (telemedicina) & $7(17,5)$ \\
Apoio psicossocial online & $3(7,5)$ \\
Vídeo educativo sobre exercícios físicos & $3(7,5)$ \\
Aplicativo para meditação & $2(5,0)$ \\
Programa de apoio online sobre estresse & $2(5,0)$ \\
Sessão online de Mindfulness & $2(5,0)$ \\
Sessão online de terapia cognitiva comportamental & $2(5,0)$ \\
Sessão online de yoga & $2(5,0)$ \\
Educação em saúde sanitária no combate ao CoVID-19 & $1(2,5)$ \\
Orientação sobre refeições saudáveis & $1(2,5)$ \\
Programa de apoio a pessoas com esclerodermia durante pandemia & $1(2,5)$ \\
Programa de apoio a pessoas com lupus durante pandemia & $1(2,5)$ \\
Rastreamento de medo fóbico & $1(2,5)$ \\
Rastreamento de puérperas e suas experiências pós-parto & $1(2,5)$ \\
Recrutamento de doadores de sangue & $1(2,5)$ \\
Sessão individualizada de Ayurveda & $1(2,5)$ \\
Tele-intervenções para manejo de diabetes & $1(2,5)$ \\
Telereabilitação com exerćícios físicos para idosos & $1(2,5)$ \\
Telereabilitação com exercícios para pessoas com coviD-19 & $1(2,5)$ \\
Terapia de aconselhamento por Software Automatizado de Mensagens & $1(2,5)$ \\
Triagem e gestão da população por suporte telefônico & $1(2,5)$ \\
Uso da prece em pacientes da UTl & $1(2,5)$ \\
Uso de telemedicina & $1(2,5)$ \\
Video psicoeducativo sobre controle de estresse & $1(2,5)$ \\
Aconselhamento financeiro & $1(2,5)$ \\
\hline
\end{tabular}

UTI- Unidade de Terapia Intensiva

\section{Discussão}

Neste estudo, identificou-se que a maioria dos experimentos eram randomizados e com alocação paralela. Esse achado é relevante, uma vez que a confiabilidade da relação causa-efeito, dos ensaios clínicos, está associada à comparação de grupos e a alocação aleatória dos participantes nos grupos, contribui para que exista maior chance de homogeneidade entre os participantes, no tocante a características que poderiam acarretar viés aos resultados. ${ }^{(6)}$ No cenário da pandemia, ensaios com estas características auxiliaram na compreensão dos efeitos de intervenções terapêuticas, em diferentes desfechos.

Outra característica verificada nos ensaios clínicos foi o cegamento, que foi ausente na maioria dos experimentos. Tal fato merece destaque, ao considerar que o cegamento consiste no desconhecimento referente à alocação de cada participante, que pode ser aplicado aos próprios participantes e/ou aos membros da equipe de pesquisa. ${ }^{(7)}$ Tal desconhecimento (cegamento) contribui para evitar surgimento, aferição ou superestimação dos efeitos observados, uma vez que os participantes podem acreditar e referir uma melhora ou piora, que não condizem com a realidade, por saberem que encontram-se no grupo que recebeu determinada intervenção. No mesmo raciocínio, os pesquisadores podem, involuntariamente, possuírem aferição tendenciosa, inclinada para os resultados que almejam encontrar, se souberem o grupo de pertencimento dos participantes. ${ }^{(7)}$

Tal situação é agravada ao considerar que o cegamento consiste em um dos critérios avaliados para que os ensaios clínicos sejam incluídos em revisóes sistemáticas. ${ }^{(8)}$ Assim, a grande quantidade de experimentos sobre a COVID-19 sem cegamento, pode constituir relevante barreira para a realização das revisóes sistemáticas e metanálises referentes à temática. Cabe destacar que as metanálises norteiam a decisão das condutas a serem incorporadas à prática clínica e, logo, são importantes para enfrentamento da pandemia.

Observou-se que a maioria dos estudos não havia iniciado o recrutamento dos participantes, para testagem das intervençóes dos ensaios clínicos. Tal fato pode ser compreendido, uma vez que o início do recrutamento depende de etapas inerentes à realização de pesquisa, como construção de projeto, autorizaçóes éticas e financiamento. ${ }^{(9)}$ Embora, no contexto da pandemia, a presteza na disponibilização de evidências científicas seja importante, o seguimento de tais etapas é relevante, por refletirem na qualidade dos resultados obtidos.

O público-alvo das intervençôes foi composto, em sua maioria, por adultos e idosos. Revisões sistemáticas apresentaram evidências de que pessoas com idade acima de 65 anos, com COVID-19, tinham maior risco de agravamento e morte e que, em crianças, a doença teve curso mais leve e melhores prognósticos do que em adultos. ${ }^{(10,11)}$ Ao comparar características clínicas de grupo de pacientes idosos com outro de jovens e adultos de meia idade, com COVID-19, estudo realizado na China identificou maior mortalidade de idosos. ${ }^{(12)}$ Essas evidências justificam a importância da condução de estudos experimentais com esses públicos, uma vez que são os mais acometidos pela infecção e mais propensos à piores desfechos. 
$\mathrm{O}$ assunto investigado em mais da metade dos ensaios clínicos, foi referente ao tratamento medicamentoso, cujo mais investigado foi a Hidroxicloroquina. Esse achado pode ser justificado, uma vez que esse medicamento, amplamente utilizado contra a malária, possui baixo custo, o que viabilizaria a sua ampla utilização para enfrentamento da pandemia, e mostrou-se eficaz no controle da infecção de COVID-19, invitro. ${ }^{(13)}$ Entretanto, resultados de duas metanálises apontaram inexistência de segurança, para a sua utilização clínica, por não possui benefício, elevar o risco de eventos adversos e aumentar a mortalidade, em pacientes com a COVID-19..$^{(14,15)}$

Entre os estudos que utilizaram intervenções biológicas, observou-se maior frequência do uso de anticorpo monoclonal humano. Estes tratam-se da principal classe de bioterapêuticos para imunoterapia passiva, no combate à infecção viral, e têm-se reconhecido como potencial tratamento para muitas doenças. ${ }^{(16)}$ Assim, resultados promissores no uso de anticorpos monoclonais para tratamento de outras formas de coronavírus (MERS-CoV e SARS-CoV), que geraram epidemia e mortes em diferentes países, tem motivado pesquisadores a desenvolverem imunoterapia baseada na associação de anticorpos monoclonais para a COVID-19. ${ }^{(17)}$

As intervençóes com plasma convalescente foram as mais utilizadas, entre os estudos experimentais com sangue e derivados. $\mathrm{O}$ uso do plasma convalescente possui histórico positivo no tratamento em outras infecçóes virais, tais como a influenza pandêmica $A(H 1 N 1)$, influenza aviária $A(H 5 N 1)$ e febres hemorrágicas, como o Ebola. ${ }^{(18)}$ No contexto da pandemia de COVID-19, esse tratamento tem sido testado e série de casos têm mostrado que pacientes com insuficiência respiratória grave, que receberam essa intervenção, apresentaram aumento dos anticorpos neutralizantes e apresentaram melhoras clínicas. ${ }^{(19)}$ No entanto, os estudos disponíveis sobre esse tipo de tratamento possuem baixo nível de evidência e a condução de estudos experimentais que esclareçam seus efeitos se fazem necessários.

Entre os estudos experimentais sobre diagnóstico da COVID-19, prevaleceram as intervençóes com uso da sorologia. O surto de infecçóes pelo SARS-CoV-2 exigiu dos países o emprego de recursos para aumento na testagem em massa da população, e, assim, as demandas na produção de testes diagnósticos foram crescentes. ${ }^{(20)}$ Nesse sentido, além de testes diretos, por meio do uso de swab de rinofaringe (RT-PCR), imunoensaios sorológicos integraram as técnicas para diagnóstico. ${ }^{(21)}$ Dessa forma, justifica-se a relevância na produção de estudos experimentais de diagnósticos sorológicos para COVID-19.

Ao considerar a importância da nutrição na recuperação da COVID-19, observou-se que a maioria dos ensaios clínicos que contemplaram tal aspecto, investigaram a suplementação das vitaminas $\mathrm{C}$ e D e do zinco. Investigaçóes nessa perspectiva são pertinentes uma vez que, a maioria dos pacientes infectados apresentam-se, na admissão hospitalar, com inflamação e anorexia graves e que há possibilidade da suplementação com esses micronutrientes contribuírem com a modulação da função imunológica e com a redução do risco de infecção. ${ }^{(22,23)}$

Em relação às intervençóes com uso de equipamentos, houve predominância daqueles relacionados ao sistema respiratório e sanguíneo. Esse resultado é esperado, uma vez que o diagnóstico e tratamento dessa doença envolve a utilização de diferentes dispositivos, sobretudo para coleta/análise de sangue e oxigenoterapia. ${ }^{(24,25)}$ Assim, ao considerar as consequências sistêmicas da COVID-19, são pertinentes os estudos que atestem a aplicabilidade e eficácia no uso desses equipamentos.

A assistência aos pacientes graves, com COVID-19, envolve cuidados para manutenção das funçôes do sistema respiratório. Entre os cuidados assistenciais, destacou-se terapia por meio da implementação da posição prona, para melhoria da eficiência pulmonar dos pacientes. Metanálise, que buscou evidência da aplicação desse posicionamento, apontou que a pronação pode diminuir a mortalidade em pacientes com síndrome respiratória aguda grave, quando aplicada nas primeiras horas de internação de pacientes com oxigenação prejudicada. ${ }^{(26)}$ Dessa forma, a investigação dos efeitos da implementação dessa posição, a melhor forma de realizá-la e os intervalos de tempo da sua utilização, 
devem ser alvo de mais estudos experimentais, para contribuir com a prática baseada em evidências dos profissionais que a realizam.

Em relação às intervenções comportamentais, observou-se predominância do monitoramento domiciliar por telemedicina. Aponta-se que tais investigaçóes podem ter sido motivadas pelas recomendações de acompanhamento profissional, sem aproximação física ou necessidade de deslocamento dos pacientes para as instituiçóes de saúde. Ademais, a telemedicina torna-se estratégia importante, diante do aumento do número de casos de contaminação pelo coronavírus SARS-CoV-2, com demanda crescente de abordagem pré-clínica, suporte assistencial e consulta. ${ }^{(27,28)}$ Nesse sentido, a escolha da tecnologia a ser utilizada poderá ser feita, mediante resultado de pesquisas que demonstrem os resultados da sua utilização.

Aponta-se como limitação do estudo a possibilidade de não inclusão de ensaios clínicos que não encontravam-se registrados nos portais pesquisados. Entretanto, aponta-se que os portais investigados possuem ampla adesão de cadastro, na realidade brasileira e, principalmente, internacional, o que é corroborado pela quantidade de ensaios encontrados e analisados no presente estudo.

A contribuiçãao deste estudo versa sobre o apontamento de lacunas, referentes aos temas, características metodológicas e localizações geográficas dos ensaios clínicos, que podem motivar e direcionar a realização de estudos, que objetivem preencher tais lacunas. Além disso, ao ser apresentada a descrição dos estudos que estão em andamento, é possível determinar a tendência das próximas publicaçôes de experimentos, que podem interessar aos profissionais da saúde e pesquisadores.

\section{Conclusão}

O panorama mundial da produção de ensaios clínicos sobre COVID-19 possui predominância de estudos realizados por instituiçóes hospitalares, oriundos da Europa, cujo recrutamento dos participantes não tinha sido iniciado, com objetivo direcionado ao tratamento e com população estudada composta por adultos e idosos, de ambos os sexos. O delineamento da maioria dos estudos incluiu randomização e alocação paralela dos participantes, mas não possuiu o cegamento. As intervenções mais estudadas foram acerca de tratamento medicamentoso, além delas, foram testadas intervençôes biológicas, com sangue e derivados, uso de equipamentos, procedimentos assistenciais, testes para diagnóstico, suplementação dietética e intervenções comportamentais. O medicamento mais estudado foi a Hidroxicloroquina, a intervenção biológica mais estudada foi com anticorpo monoclonal, a intervenção com sangue e derivados mais pesquisada foi com plasma convalescente, a intervenção diagnóstica foi a por sorologia, os equipamentos mais estudados versavam sobre os voltados aos sistemas respiratório e sanguíneo, o procedimento assistencial que mais foi alvo dos experimentos foi o posicionamento em prona, a intervenção nutricional foi a suplementação das Vitaminas C e D e do Zinco e a intervençáo comportamental mais estudada foi o monitoramento domiciliar por telemedicina. Para disponibilização de evidências científicas que respaldem as decisóes clínicas e com vistas contribuir para avanço do conhecimento sobre a COVID-19, recomenda-se a realização de estudos que contemplem as lacunas identificadas, referentes às pesquisas sobre o contexto da América, Ásia, África e Oceania; realizadas com recém-nascidos, crianças e adolescentes; que investiguem a prevenção, diagnóstico, reabilitação e aspectos referentes à saúde mental e que, no seu desenho metodológico, possuam cegamento.

\section{Colaborações}

Barros LM participou da concepção e projeto, coleta e interpretação dos dados, redação do artigo, revisão crítica relevante do conteúdo intelectual e aprovação final da versão a ser publicada. Galindo Neto NM e Sá GGM participaram da análise e interpretação dos dados, redação do artigo, revisão crítica relevante do conteúdo intelectual e aprovação final da versão a ser publicada. Pereira JCN, Pereira JCN, Barbosa LU, Oliveira Neto JGO, Henriques AHB e Caetano JÁ participaram da revisão crítica rele- 
vante do conteúdo intelectual e aprovação final da versão a ser publicada e concordância com todos os aspectos do manuscrito em termos de veracidade ou integridade das informaçóes.

\section{Referências}

1. World Health Organization (WHO). Cononavirus disease (COVID-19) Situation Report - 127 [Internet]. Geneva: WHO; 2020. [cited 2020 May 18]. Available from: https://www.who.int/emergencies/diseases/ novel-coronavirus-2019/situation-reports

2. Lai CC, Shih TP, Ko WC, Tang HJ, Hsueh PR. Severe acute respiratory syndrome coronavirus 2 (SARS-CoV-2) and coronavirus disease-2019 (COVID-19): the epidemic and the challenges. Int J Antimicrob Agents. 2020;55(3):105924.

3. Tu YF, Chien CS, Yarmishyn AA, Lin YY, Luo YH, Lin YT, et al. A Review of SARS-CoV-2 and the Ongoing Clinical Trials. Int J Mol Sci. 2020;21(7):2657.

4. Lu H. Drug treatment options for the 2019-new coronavirus (2019nCoV). Biosci Trends. 2020;14(1):69-71.

5. Wu Z, McGoogan JM. Characteristics of and important lessons from the coronavirus disease 2019 (COVID-19) Outbreak in China: Summary of a Report of 72314 cases from the chinese Center for Disease Control and Prevention. JAMA. 2020 Feb 24. doi: 10.1001/ jama.2020.2648.

6. Bouwmeester $\mathrm{S}$, Jongerling J. Power of a randomization test in a single case multiple baseline AB design. PLoS One. 2020;15(2):e0228355.

7. Braithwaite FA, Walters JL, Li LS, Moseley GL, Williams MT, McEvoy MP. Effectiveness and adequacy of blinding in the moderation of pain outcomes: systematic review and meta-analyses of dry needling trials. PeerJ. 2018;6(6):e5318.

8. Bello S, Moustgaard H, Hróbjartsson A. Unreported formal assessment of unblinding occurred in 4 of 10 randomized clinical trials, unreported loss of blinding in 1 of 10 trials. J Clin Epidemiol. 2017;81:42-50.

9. Batagello R. Recruitment of subjects in industry-sponsored multicenter international clinical trials. Saude Soc. 2018;27(4):1033-43.

10. Zheng Z, Peng F, Xu B, Zhao J, Liu H, Peng J, et al. Risk factors of critical \& mortal COVID-19 cases: A systematic literature review and meta-analysis. J Infect. 2020;81(2):e16-25.

11. Ludvigsson JF. Systematic review of COVID-19 in children shows milder cases and a better prognosis than adults. Acta Paediatr. 2020;109(6):1088-95.

12. Liu K, Chen Y, Lin R, Han K. Clinical features of COVID-19 in elderly patients: A comparison with young and middle-aged patients. J Infect. 2020;80(6):e14-8.

13. Wang M, Cao R, Zhang L, Yang X, Liu J, Xu M, et al. Remdesivir and chloroquine effectively inhibit the recently emerged novel coronavirus (2019-nCoV) in vitro. Cell Res. 2020;30(3):269-71.
14. Sarma P, Kaur H, Kumar H, Mahendru D, Avti P, Bhattacharyya A, et al. Virological and clinical cure in COVID-19 patients treated with hydroxychloroquine: A systematic review and meta-analysis. J Med Virol. 2020;92(7):776-85.

15. Singh AK, Singh A, Singh R, Misra A. "Hydroxychloroquine in patients with COVID-19: A Systematic Review and meta-analysis.". Diabetes Metab Syndr. 2020;14(4):589-96.

16. Mire CE, Chan YP, Borisevich V, Cross RW, Yan L, Agans KN, et al. A cross-reactive humanized monoclonal antibody targeting fusion glycoprotein function protects ferrets against lethal nipah virus and hendra virus infection. J Infect Disease. 2020;221(Supl 4):S471-S479.

17. Shanmugaraj B, Siriwattananon K, Wangkanont K, Phoolcharoen W. Perspectives on monoclonal antibody therapy as potential therapeutic intervention for Coronavirus disease-19 (COVID-19). Asian Pac J Allergy Immunol. 2020;38(1):10-8.

18. Dean CL, Hooper JW, Dye JM, Zak SE, Koepsell SA, Corash L, et al. Characterization of Ebola convalescent plasma donor immune response and psoralen treated plasma in the United States. Transfusion. 2020;60(5):1024-31.

19. Duan K, Liu B, Li C, Zhang H, Yu T, Qu J, et al. Effectiveness of convalescent plasma therapy in severe COVID-19 patients. Proc Natl Acad Sci USA. 2020;117(17):9490-6.

20. Pan Y, Li X, Yang G, Fan J, Tang Y, Zhao J, et al. Serological immunochromatographic approach in diagnosis with SARS-CoV-2 infected COVID-19 patients. J Infect. 2020;81(1):e28-32.

21. Sanduzzi A, Zamparelli SS. Nasopharyngeal and oropharyngeal swabs, and/or serology for SARS COVID-19: what are we looking for? Int J Environ Res Public Health. 2020;17(9):e3289.

22. Caccialanza R, Laviano A, Lobascio F, Montagna E, Bruno R, Ludovisi S, et al. Early nutritional supplementation in non-critically ill patients hospitalized for the 2019 novel coronavirus disease (COVID-19): rationale and feasibility of a shared pragmatic protocol. Nutrition. 2020;74:110835.

23. Gombart AF, Pierre A, Maggini S. A review of micronutrients and the immune system-working in harmony to reduce the risk of infection. Nutrients. 2020;12(1):236.

24. Li Z, Liu G, Wang L, Liang Y, Zhou Q, Wu F, et al. From the insight of glucose metabolism disorder: oxygen therapy and blood glucose monitoring are crucial for quarantined COVID-19 patients. Ecotoxicol Environ Saf. 2020;197:110614.

25. Guo L, Ren L, Yang S, Xiao M, Chang D, Yang F, et al. Profiling Early Humoral Response to Diagnose Novel Coronavirus Disease (COVID-19). Clin Infect Dis. 2020;71(15):778-85.

26. Munshi L, Del Sorbo L, Adhikari NKJ, Hodgson CL, Wunsch H, Meade $\mathrm{MO}$, et al. Prone position for acute respiratory distress syndrome. A systematic review and meta-analysis. Ann Am Thorac Soc. 2017;14(Suppl 4): S280-S288.

27. Sarti TD, Lazarini WS, Fontenelle LF, Almeida AP. What is the role of Primary Health Care in the COVID-19 pandemic? Epidemiol Serv Saude. 2020;29(2):e2020166.

28. Hong Z, Li N, Li D, Li J, Li B, Xiong W, et al. Telemedicine During the COVID-19 Pandemic: Experiences From Western China. J Med Internet Res. 2020;22(5):e19577. 